\title{
Unidentified Species in Envelopes around Carbon Stars
}

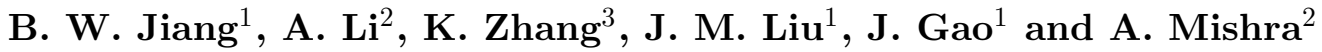 \\ ${ }^{1}$ Department of Astronomy, Beijing Normal University, Beijing 100875, China \\ email:bjiang@bnu.edu.cn \\ ${ }^{2}$ Department of Physics and Astronomy, University of Missouri, Columbia, MO 65211, USA \\ ${ }^{3}$ Department of Physics, California Institute of Technology, Pasadena, CA 91125, USA
}

\begin{abstract}
The infrared (IR) spectra of many evolved carbon-rich stars exhibit two prominent dust emission features peaking around $21 \mu \mathrm{m}$ and $30 \mu \mathrm{m}$, with the former exclusively seen in proto-planetary nebulae (PPNe), while the latter seen in a much wider range of objects, including AGB stars, PPNe and planetary nebulae (PNe). The $30 \mu \mathrm{m}$ feature is seen in all the $21 \mu \mathrm{m}$ sources, but no correlation is found between these two features. Over a dozen carrier candidates have been proposed for the $21 \mu \mathrm{m}$ feature, but none of them has been widely accepted and the nature of the $21 \mu \mathrm{m}$ feature remains a mystery. The carrier of the $30 \mu \mathrm{m}$ feature also remains unidentified. MgS dust, once widely accepted as a valid carrier, was ruled out because of the sulfur budget problem. In this work we examine nano-sized $\mathrm{FeO}$ dust as a carrier for the $21 \mu \mathrm{m}$ feature. We calculate the IR emission spectrum of $\mathrm{FeO}$ nanodust which undergoes single-photon heating in PPNe. It is found that the $21 \mu \mathrm{m}$ feature emitted by $\mathrm{FeO}$ nanodust is too broad to explain the observed feature. For the $30 \mu \mathrm{m}$ feature, we argue that graphite could be a viable

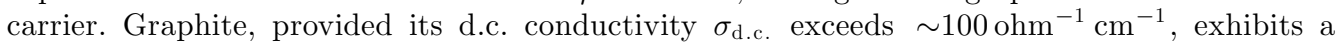
pronounced band at $30 \mu \mathrm{m}$.
\end{abstract}

Keywords. stars: carbon, (stars:) circumstellar matter, dust, infrared: stars

\section{Introduction}

In the late stage of evolution, the low- and intermediate-mass stars lose a large amount of mass and produce circumstellar shells. Various dust species are present in the cold and dense shells of asymptotic giant branch (AGB) stars, post-AGB stars and planetary nebulae (PNe), among which silicate dust in O-rich shells and $\mathrm{SiC}$ dust in C-rich shells are well identified through their spectral features.

There are two prominent spectral features respectively peaking around $21 \mu \mathrm{m}$ and $30 \mu \mathrm{m}$. Both features are observed in circumstellar shells of C-rich stars in both the Milky Way and the Magellanic Clouds (MC). So far, the $21 \mu \mathrm{m}$ feature is detected in about 18 Galactic objects (Cerrigone et al. 2011) and 9 MC objects (Volk et al. 2011). The $21 \mu \mathrm{m}$ feature is only seen in proto-planetary nebulae (PPNe), a transient phase in stellar evolution. In contrast, the $30 \mu \mathrm{m}$ feature is detected in the whole late stage of stellar evolution, from AGB to post-AGB to PN (Hony et al. 2003).

All the $21 \mu \mathrm{m}$ sources also display the $30 \mu \mathrm{m}$ emission feature as well as the so-called "unidentified infrared (UIR)" emission bands at 3.3, 6.2, 7.7, 8.6 and $11.3 \mu \mathrm{m}$. The UIR bands are commonly attributed to polycyclic aromatic hydrocarbon (PAH) molecules (Léger \& Puget 1984, Allamandola et al. 1985). As shown in Figure 1, the $30 \mu \mathrm{m}$ feature does not correlate with the $21 \mu \mathrm{m}$ feature, implying that their carriers are not related. Figure 1 also shows that the $21 \mu \mathrm{m}$ feature does not correlate with the UIR features. This argues against large PAH clusters as a possible carrier for the $21 \mu \mathrm{m}$ feature. Moreover, it appears that the $30 \mu \mathrm{m}$ feature and the UIR features weakly anti-correlate, suggesting 

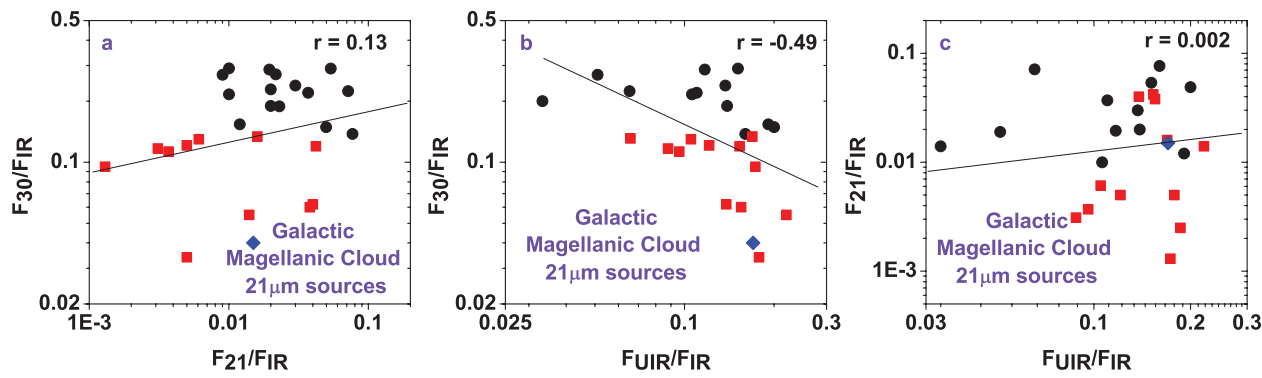

Figure 1. Interrelations among the $21 \mu \mathrm{m}, 30 \mu \mathrm{m}$, and UIR features of Galactic (circles) and MC (squares) $21 \mu \mathrm{m}$ sources: (a) $F_{30 \mu \mathrm{m}}$ vs. $F_{21 \mu \mathrm{m}}$, (b) $F_{30 \mu \mathrm{m}}$ vs. $F_{\mathrm{UIR}}$, and (c) $F_{21 \mu \mathrm{m}}$ vs. $F_{\mathrm{UIR}}$. All quantities are normalized by $F_{\text {IR }}$, the total near- to mid-IR emission obtained by $I S O / \mathrm{SWS}$ in the 2-45 $\mu \mathrm{m}$ wavelength range, to cancel out their common proportionality to $F_{\mathrm{IR}}$ (i.e. the illuminating starlight intensity and the bulk dust quantity).
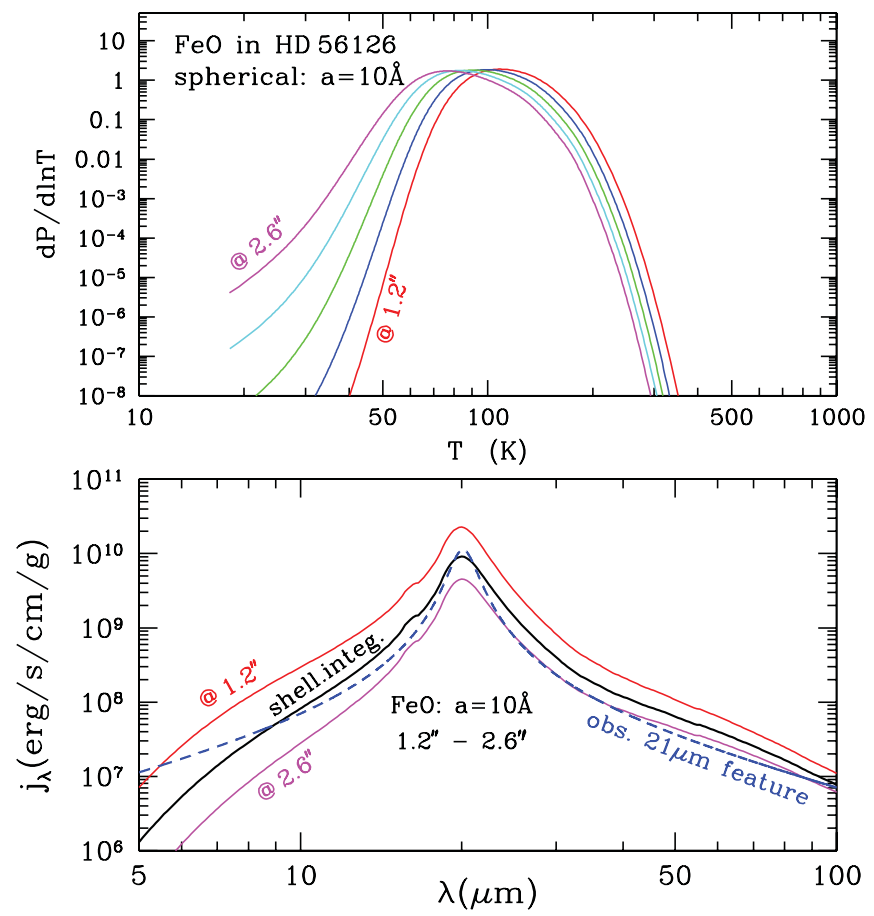

Figure 2. Upper panel: the temperature probability distribution functions $d P / d T$ of $\mathrm{FeO}$ dust of $a=1 \mathrm{~nm}$ at various distances from the illuminating star HD 56126. Lower panel: The emission spectra of $\mathrm{FeO}$ dust of $a=1 \mathrm{~nm}$ at the inner and outer shells (i.e., 1.2 $2^{\prime \prime}$ and $2.6^{\prime \prime}$ from the central star), as well as that obtained from integrating the whole dust shell. Also shown is the observed $21 \mu \mathrm{m}$ emission feature.

that the UIR carriers (e.g. PAHs) may result from the decomposition or shattering of the $30 \mu \mathrm{m}$-feature carrier.

\section{FeO nanodust as a carrier of the $21 \mu \mathrm{m}$ feature}

Zhang et al. (2009a) examined nine inorganic carrier candidates for the $21 \mu \mathrm{m}$ feature, including nano $\mathrm{TiC}$, fullerenes with $\mathrm{Ti}, \mathrm{SiS}_{2}$, doped $\mathrm{SiC}$, the $\mathrm{SiC}$ and $\mathrm{SiO}_{2}$ mixture, $\mathrm{FeO}$, $\mathrm{Fe}_{2} \mathrm{O}_{3}$ and $\mathrm{Fe}_{3} \mathrm{O}_{4}$. We found that except $\mathrm{FeO}$ nanodust, they are all problematic: they 

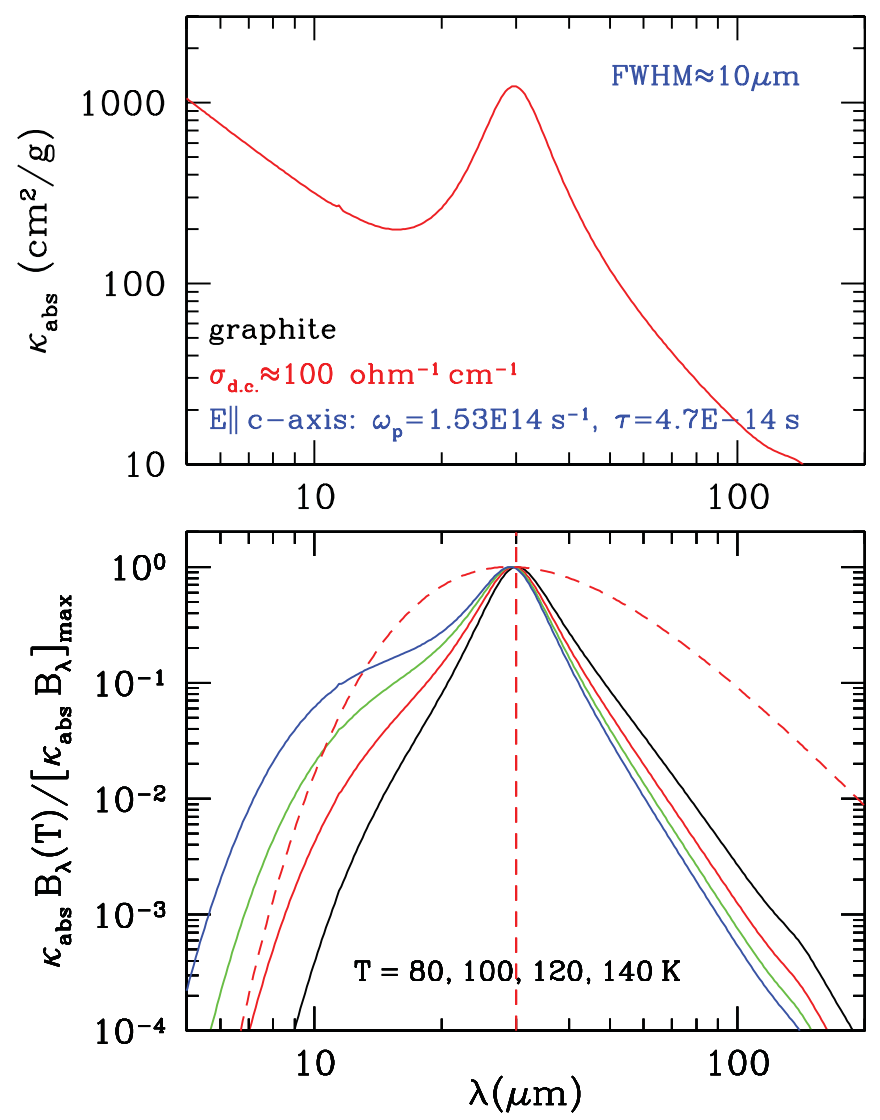

Figure 3. Upper panel: the opacity of graphite with a d.c. conductivity of $\sigma_{\text {d.c. }}=100 \mathrm{ohm}^{-1} \mathrm{~cm}^{-1}$. A prominent $30 \mu \mathrm{m}$ feature with a FWHM of $\sim 10 \mu \mathrm{m}$ is clearly seen. Lower panel: the emission profiles of graphite with $\sigma_{\text {d.c. }}=100 \mathrm{ohm}^{-1} \mathrm{~cm}^{-1}$ at $T=80,100$, 120, $140 \mathrm{~K}$. Dot-dashed line: the $T=100 \mathrm{~K}$ blackbody.

either require too much material (i.e., violating the abundance constraint) or produce extra features which are not seen in astronomical objects.

$\mathrm{FeO}$ seems to be a promising candidate carrier for the $21 \mu \mathrm{m}$ feature for two reasons: (1) Fe is an abundant element; and (2) FeO has a pronounced spectral feature around $21 \mu \mathrm{m}$ (Posch et al. 2004, Zhang et al. 2009a). However, it is not clear how FeO forms in C-rich environments as the $21 \mu \mathrm{m}$ sources are all C-rich. Posch et al. (2004) argued that $\mathrm{FeO}$ nanodust could form in these environments. We therefore model the excitation and emission processes of $\mathrm{FeO}$ nanodust in PPNe. As $\mathrm{FeO}$ nanodust will experience temperature fluctuation, we calculate its temperature probability distribution function $d P / d T$ and then calculate its emission spectrum from $d P / d T$.

Taking a 3-D Debye model (with a Debye temperature of $\Theta_{\mathrm{D}}=430 \mathrm{~K}$ ) for the specific heat of $\mathrm{FeO}$ and the refractive indices at $\mathrm{T}=10 \mathrm{~K}, 100 \mathrm{~K}, 200 \mathrm{~K}$ and $300 \mathrm{~K}$ from Henning $\&$ Mutschke $(1995,1997)$, we calculate $d P / d T$ and the emergent spectrum of spherical FeO of radius $a=10 \AA$ for HD 56126, a proto-typical $21 \mu \mathrm{m}$ source (see Figure 1). The IR emission spectrum exhibits a strong band at $21 \mu \mathrm{m}$. However, it is too broad (with FWHM $\sim 4 \mu \mathrm{m}$ ) to explain the observed FWHM of $\sim 2.2-2.3 \mu \mathrm{m}$ (see Kwok et al. 1989). For nonspherical dust, the predicted $21 \mu \mathrm{m}$ feature is even broader. Finally, to account for the power emitted from the $21 \mu \mathrm{m}$ feature in HD 56126, the FeO model 
requires $\mathrm{Fe} / \mathrm{H} \approx 4.3 \times 10^{-6}$, exceeding the iron budget of $\mathrm{Fe} / \mathrm{H} \approx 3.2 \times 10^{-6}$ available in HD 56126. Therefore, FeO nanodust is unlikely a valid carrier for the $21 \mu \mathrm{m}$ feature.

\section{Graphite as a carrier for the $30 \mu \mathrm{m}$ feature}

The $30 \mu \mathrm{m}$ feature, extending from $\sim 24$ to $\sim 45 \mu \mathrm{m}$, is very strong and accounts for up to $\sim 30 \%$ of the total IR luminosity of the emitter. MgS dust, once widely accepted as a carrier (Begemann et al. 1994), was ruled out by Zhang et al. (2009b) who showed that the MgS model would require too much S. Even with a very generous assumption of $\mathrm{UV} /$ optical absorbing coefficient, the required MgS dust exceeds what is available by over an order of magnitude. This was later confirmed by Messenger et al. (2013) (but also see Lombaert et al. 2012).

We propose graphite as a carrier of the $30 \mu \mathrm{m}$ feature seen in evolved stars. Graphite has been identified as a presolar stardust species in primitive meteorites through its isotropic anomaly. Graphite is also key component in standard interstellar grain models. A feature around $30 \mu \mathrm{m}$ is already recognizable in the absorption coefficient of graphite (Draine \& Lee 1984). Depending on its d.c. conductivity $\sigma_{\text {d.c. }}$, the $30 \mu \mathrm{m}$ feature could be very strong. Our preliminary calculations show that graphite could account for the observed $30 \mu \mathrm{m}$ feature, provided $\sigma_{\text {d.c. }}>100 \mathrm{ohm}^{-1} \mathrm{~cm}^{-1}$ (see Figure 1). Experiments have shown that graphite can have a wide range of $\sigma_{\text {d.c. }}$, ranging from $\sim 1$ to $\sim 200 \mathrm{ohm}^{-1} \mathrm{~cm}^{-1}$ (Primak 1956).

\section{Acknowledgements}

We are supported in part by NSF AST-1109039, NNX13AE63G, NSFC 11173007, NSFC 11173019, and the University of Missouri Research Board.

\section{References}

Allamandola, L. J., Tielens, A. G. G. M., \& Barker, J. R. 1985, ApJ, 290, L25

Begemann, B., et al. 1994, ApJ, 423, L71

Cerrigone, L., Hora, J. L., Umana, G., Trigilio, C., Hart, A., \& Fazio, G. 2011, ApJ, 738, 121

Draine, B. T. \& Lee, H. M. 1984, ApJ, 285, 89

Henning, T. \& Mutschke, H. 1995, A\&SAS, 112, 143

Henning, T. \& Mutschke, H. 1997, A\&SA, 327,743

Hony, S., et al. 2003, A\&BA, 402, 211

Kwok, S., Volk, K. M., \& Hrivnak, B. J. 1989, ApJ, 345, L51

Léger, A. \& Puget, J. 1984, A\&SA, 137, L5

Lombaert, R., de Vries, B. L., de Koter, A., et al. 2012, A\&BA, 544, L18

Messenger, S., Speck, A., \& Volk, K. 2013, ApJ, 764, 142

Posch, Th., Mutschke, H., \& Andersen, A. 2004, ApJ, 616, 1167

Primak, W. 1956, Phys. Rev., 103, 544

Volk, K., Hrivnak, B. J., Matsuura, M., et al. 2011, ApJ, 735, 127

Zhang, K., Jiang, B. W., \& Li, A. 2009a, MNRAS, 396, 1247

Zhang, K., Jiang, B. W., \& Li, A. 2009b, ApJ, 702,680 